\title{
Iniciativas de gestión pública que promueven la productividad desde una perspectiva del género
}

\author{
Cristian Eugenio Orellana Pino \\ Universidad Diego Portales \\ Hernán José Perotti \\ Universidad Nacional del Litoral
}

\begin{abstract}
Este trabajo es un análisis crítico de la gestión organizacional que estudia la participación de la mujer en formatos productivos locales en dos regiones: la provincia de Santa Fe en Argentina y en las regiones rurales de la Zona Central de Chile. Ambos análisis exponen una visión acerca del riesgo del retorno de la inversión del capital socio-económico en formatos productivos de desarrollo local que son afectados por intervenciones de la administración de políticas públicas en el ámbito de la mujer. Este trabajo constituye un análisis cualitativo descriptivo y de proceso heurístico sobre la visión que se tiene del género, y particularmente de la mujer, para entender qué tipos iniciativas estimulan la participación hacia esa forma de organización de trabajo de coproducción y bajo qué condiciones estas ocurren.
\end{abstract}

Palabras clave: intervenciones políticas; formatos productivos locales; género; mujer.

Iniciativas de gestão pública que promovem a produtividade a partir de uma perspectiva do gênero

Este trabalho é uma análise crítica da gestão organizacional que estuda a participação da mulher nos arranjos produtivos locais em duas regiões: a província de Santa Fe, Argentina, e os setores rurais na Zona Central do Chile. Ambos os casos expõem uma visão do risco do retorno da inversão do capital econômico e social em arranjos produtivos locais que são afetados por intervenções da gestão das políticas públicas no âmbito da mulher. Este trabalho constitui uma análise qualitativa descritiva e de processo heurístico sobre a visão que se tem do gênero, e particularmente da mulher, para compreender que tipos de iniciativas incentivam a participação nessa forma de organização do trabalho de coprodução e sob que condições estas ocorrem.

Palavras chave: intervenção política local; arranjos produtivos locais; gênero; mulher.

http://dx.doi.org/10.1590/0034-76121028

Artículo sometido en 11 feb. 2012 y aceptado en 18 dic. 2013.

Rev. Adm. Pública - Rio de Janeiro 48(2):507-528, mar./abr. 2014 
Public management initiatives to promote gender productivity

This work is proposed as a phenomenological analysis of critical organizational management which examines gender participation in local production in two regions of neighboring countries: the province of Santa Fe in Argentina and the Central Region of Chile. Both cases develop the vision of risk that exists on economic and social capital investment results when local productive sectors are affected by public policy interventions and initiatives, specially when they are implemented with a focus on women and the phenomenon of gender participation. An heuristic qualitative study is developed as a methodology to analyze the phenomenon of gender to understand what kind of initiatives to encourage participation on this aspect of organization may be implemented and under what conditions they occur.

KEYWORDs: political intervention; local co-productive formats; gender; woman.

\section{Preámbulo a la gestión pública}

El análisis del presente trabajo se dota de un estudio cualitativo descriptivo que constata la realidad de dos regiones cuyos componentes administrativos-políticos y económico-regionales comparten su naturaleza de estar insertas en un proceso de políticas de acción afirmativa en la agenda de gobierno. En ambos casos coexisten acciones de cuerpo político-administrativos de gobiernos democráticos cuyas políticas públicas sociales se orientan a desarrollar programas económicos con estructura de base social y que a su vez tienen su principal foco en la representación de la mujer como fenómeno de género, particularmente en condiciones de pobreza y exclusión laboral. Cabe destacar que este análisis excluye el efecto referencial que producen normalmente en algunos países sub-desarrollados estos tipos de políticas públicas: la generación de dependencias y la proyección del carácter asistencialista. Se establece como matriz de estudio la naturaleza del ambiente y entorno que tiene el desarrollo innovador local, a la vez que hemos considerado algunas variables fundamentales para entender el estado del arte, para lograr la modernidad en la gestión pública, que entreguen soluciones oportunas y que sean eficaces.

Para el análisis de las gobernanzas locales, observamos dos perfiles de administración: el descentralizado en la provincia de Santa Fe (Argentina) y el segundo centralizado en los sectores urbano-rurales en la Zona Central de Chile. Es posible constatar que en ambos casos se logran consolidar sus acciones de programas de reconversión económica social por medio de disímiles estrategias de desarrollo local que actúan in locus. Cada gobierno también fomenta de manera indirecta sus programas de protección social, sea a través de la autogestión en la creación de emprendimientos locales para cada región (orientando sus acciones hacia la entrega de recursos y herramientas de gestión) como también brindando capacitaciones a mujeres en situaciones de vulnerabilidad económica y social. La diferencia principal radica en la responsabilidad en las líneas presupuestarias que poseen ambos gobiernos hacia estas acciones, y en las responsabilidades de sobre quién recae esta gestión y el mecanismo de distribución de los recursos económicos. Los gobiernos provinciales, como es el caso de la Provincia de Santa Fe, hacen valer su autodeterminación al sobreponer sus políticas locales ante el gobierno central, cuya legalidad es la consistencia entre sus programas de desarrollo y la autonomía local. 
Para el caso chileno, que posee un estado de políticas centralizadas, se proponen programas de protección social con una fuerte orientación a la inserción laboral en sectores nichos de mercado que luego generan resultados positivos ante las limitadas opciones que ofrece este particular sistema. Ambos sistemas de organización presentan mecanismos políticos volcados hacia las minorías que participan de estos programas, donde pueden revelar sus problemas en un plano local y orientar los intereses ante una mayoría centralizada, sin dejar de lado la manera de encaminar las políticas de cooperación para acotar el gasto fiscal.

Para el caso federativo de la organización provincial de Santa Fe existe una superposición de intereses centralizados con los subnacionales, que puede opacar el potencial que las regiones pueden aportar hacia la mayoría de las provincias. En este aspecto existe un acción que neutraliza los intereses locales frente a la imposición que crean las demandas de las esferas de gobierno central por orientar sus programas hacia otro tipo de ejercicio político (Arretche, 2004; Abrucio, 2005; Machado, 2008). Todavía existe, según Machado (2008) una imposición o sutil inconsistencia en la literatura teórica respecto a cuál es la forma correcta de organización de un Estado orientado hacia programas de protección social, sin producir efectos nocivos en ambos sistemas, como aquella que presentan las institucionalidades atomizadas en la centralización de las acciones políticas federativas. Esta superposición de entidades gubernamentales suele afectar muchas veces a las limitadas opciones que tienen los estados subnacionales que ejercen un cierto control autonómico en sus regiones; por ejemplo: la aprobación de leyes presupuestarias locales, el ordenamiento urbanístico, el efecto de las leyes orgánicas en los estados federativos, institucionalización de normas municipales, regulación de la explotación de recursos naturales, distribución de royalties para la extracción de recursos productivos con beneficio local, que en suma permitirán una adecuada orientación del presupuesto anual.

Los mecanismos para regular estas articulaciones se construyen sobre ciertas estructuras políticas que promueven incentivos para el desarrollo productivo local. Son mecanismos adecuados que operan sobre un abanico de opciones políticas sociales que cada gobierno federativo o local toma para dichas aplicaciones en sectores de mayor necesidad económica y los sectores de mayor vulnerabilidad. Por otra parte, además de entregar una adecuada orientación en la ejecución en sus acciones pueden generar externalidades negativas en la aplicación de las políticas públicas de incentivo al desarrollo local. Por ejemplo, el hecho de persuadir el verdadero fin de la implementación de políticas locales para incrementar el número de stakeholder locales como contingente electoral o provocar una sinergia de proyectos locales de manera de aumentar el incentivo a la adhesión de oportunidades.

En relación a lo anterior, según Machado (2008) los incentivos a las oportunidades para el diseño de proyectos orientados al género hacia la mujer suelen inclinarse hacia la arbitrariedad, por ejemplo, al adoptar direcciones incorrectas en el incentivo de las políticas desde su propósito principal. Por otro lado, estos proyectos suelen incluir sanciones negativas a determinadas opciones como: aplicación excesiva de multas para conductas no adecuadas al buen comportamiento fiscal responsable de la actividad presupuestaria. Puede citarse el caso en el que incurren los administradores regionales cuando se apartan del manejo contable esperado que 
afecta la política fiscal o generan desordenes en la distribución u organización de fondos con destino al presupuesto municipal.

Lo mencionado anteriormente puede ser visto como una oportunidad creativa: las sanciones financieras por el mal uso del presupuesto municipal en las políticas públicas provocan lentitud en los procesos de entrega de fondos y en la forma de cómo deben ser implementadas las políticas, por lo que debe ser tenido en cuenta dicho efecto. Para Fernandez y Rainey (2006) esto demuestra la desconexión de los agentes y actores involucrados en la ejecución de los proyectos orientados a las políticas de género. Por ejemplo, la fuerte adhesión a políticas sociales de actores en localidades o regiones demandan un cambio en las responsabilidades de quienes hacen de la gestión un negocio particular y vuelven necesario indicar qué elementos desencadenan la lentitud y entraban los procesos de entrega de fondos económicos.

Los elementos que están contenidos y atienden al interés y grado de inversión de políticas públicas son aquellos que aportan a la generación de nuevos puestos de trabajo y no a su mantención, la búsqueda del auto-empleo o la dependencia laboral. Lo anterior queda resaltado aún más cuando el análisis se basa en aquellas motivaciones que despiertan las oportunidades laborales para las mujeres. La inversión en desarrollo del empleo es un medio para encontrar el espacio en el cual aplicar los conocimientos y desarrollar las respectivas competencias de los individuos que se encuentran limitados por el crecimiento exógeno y desarrollo del auto-empleo. No obstante, muchas veces son las propias organizaciones quienes deben dar ayuda a los individuos a encontrar e identificar los nuevos tipos y competencias que estos demandan, como además estos pueden localizar sus propias motivaciones por medio de sus propias relaciones de trabajo (Kuvaas y Dysvik, 2009).

Según Grant (2008) la solidez de una motivación es una oportunidad de fortaleza para producir relaciones entre las acciones pro sociales y los resultados en un trabajo. Y tales elementos motivacionales serían aquellas relaciones de persistencia, productividad y obtención de un eficiente desempeño. Estas acciones pro sociales son más autónomas cuando las motivaciones son altamente intrínsecas, o sea, motivadas por el sentimiento de obtención de empleo eficientemente bien desempeñado, así como el desarrollo de sus propios objetivos y metas. Lo anterior sustenta que la búsqueda de una actividad que brinde una satisfacción de autodesarrollo puede generar valores positivos y resultados que pueden ayudar a otros.

Una aplicación que es posible llevar a efecto, es la aplicación de un benchmarking externo a los formatos productivo locales como resultado de iniciativas de éxito en ambas regiones, la implementación de políticas de protección social en poblaciones de mayor riesgo salubre, el contenido en proyectos que defiendan los derechos universales de la mujer, el incentivo a proyectos de corte con generación del empleo a la mujer joven, redes productivas y generación de cluster con propiedades femeninas.

Si las políticas públicas apoyan a los programas de la mujer por la simple erradicación de elementos que deterioran el desarrollo de nuevos atractivos productivos para la inversión financiera, estas tendrán poco sustento como argumento económico. O sea, se trata de políticas áridas. Según Turok (2008) al indagar sobre estos tipos de políticas económicas en países en desarrollo los emprendimientos sufrirán siempre los estigmas de sectores excluidos. Y ocurre que las políticas se han vuelto cada vez más una oferta de recursos mal orientados por 
defectos en el diseño de los proyectos y su débil argumento de justificar las acciones y objetivos; obteniendo como resultado que se vuelvan menos atractivos los espacios para inversión privada, que se reduzca la oferta de mano de obra potencial, que se impongan altos costos para la cartera de inversión pública para aplicar programas sociales, etc.

Aún así en regiones rurales en situaciones de riesgo o vulnerabilidad, se ha visto que las políticas públicas de mercado que se aplican a necesidades locales, en poblaciones bajo una situación de pobreza es posible entregar alternativas y lograr salidas a la búsqueda de empleos con base tecnológica. (Simani y Hart, 2006). Por ejemplo, se dan casos donde las necesidades locales del tipo domésticas como la generación de electricidad, el suministro de agua potable, son la base precursora de la obtención de recursos productivos y la generación de productos de base tecnológica. De acuerdo con Kelman (2004) los gobiernos necesitan realizar mudanzas en la formas de trabajo y en la forma de hacer nuevos gobiernos de acuerdo a nuevas matrices de resultados para buenos desempeños en la generación de productos y no mecanismo o procesos de obtención de empleos. Es cierto que moviéndose de acuerdo a los cambios es que se generan las nuevas demandas en torno a las nuevas formas de gobernar.

Por ejemplo, todavía existe una fijación en las políticas de mercado como sistemas operacionales de la eficiencia de los gobiernos centralizados como ocurre en la Zona Central en Chile, y que parecen asemejarse a la administración del sector privado en la naturaleza desmedida de la búsqueda de resultados, desempeño y logro de sus programas bajo un sistema de eficiencia perfecta.

En el caso de la Provincia de Santa Fe, las políticas públicas parecieran no generar cambios productivos, y de generarlos estos se realizan con mejorías incompletas y soluciones paliativas de corto plazo por el tipo de inversión. Los programas sociales y enfocados hacia la mujer con frecuencia atribuyen sus acciones de trabajo a ejercicios rutinarios que se heredan en las gestiones que se preceden. En teoría, el logro es evidente al visualizar los cambios, convicción de que los resultados se tornen concretos y medibles. La resistencia al cambio genera descontentos e insatisfacción, sin embargo, de existir una acción o interés dispuesto es posible que dichos cambios se produzcan con menor insatisfacción. Por ejemplo, en algunas regiones de la Zona Central de Chile el sistema tradicional es una de las mayores fuentes de insatisfacción y se debe a la falta de autonomía en la toma de decisiones de interés en las representaciones locales, donde sus líderes políticos acaban delegando sus directrices a un gobierno central. Esto se explica por la excesiva carga de reglas y normas que deben soportar los funcionarios públicos, como la verticalidad de una jerarquía elitista que producen las tradicionales formas de gobiernos centralizados (Crandall y Beasley, 2003; Blaug, 2009). Además del aumento creciente de sistemas burocráticos que regulan en exceso provocando una sobrecarga y estrés laboral.

En suma, existe el descontento social que genera fuentes de insatisfacción en sistemas tradicionales de hacer la gestión de administrar y esto suele ser común tanto en la Provincia de Santa Fe como en las regiones rurales de la Zona Central de Chile. Sin embargo, en los últimos años se han efectuado esfuerzos por torcer esta tendencia mediante descentralización, particularmente en el ámbito territorial de la provincia de Santa Fe, la que administrativamente ha sido dividida en cinco regiones, que permite mediante un planificado programa de asistencia atender a las demandas y exigencias de un territorio que es heterogéneo, con- 
solidando una propuesta de solidaridad e integración en la que se fortalece la participación ciudadana en el plano local que es la creación del Plan Estratégico Provincial formulado en la Provincia de Santa Fe (2008).

El efecto multiplicador que genera una gestión pública que promueva e incorpore principios de calidad y los integre a los proyectos socio-económicos con enfoque al desarrollo local hacia la mujer, ya estimula iniciativas de movilización de recursos financieros creando una conciencia territorial de empoderamientos y aprendizajes para generar iniciativas locales. Estas acciones de empoderamientos son obtenidas de la experiencia local que aportan y adicionan valor a una cadena de procesos socio-económicos para un mejor estilo de gobernanza. En la figura 1 el mapa presenta un modelo de integración para organizaciones que desean establecer en localidades o regiones sus negocios por medio de iniciativas locales como emprendimientos que tienen como concepto el empoderamiento femenino, orientados por políticas locales y regionales.

Figura 1

Mapa de iniciativa para un desarrollo local

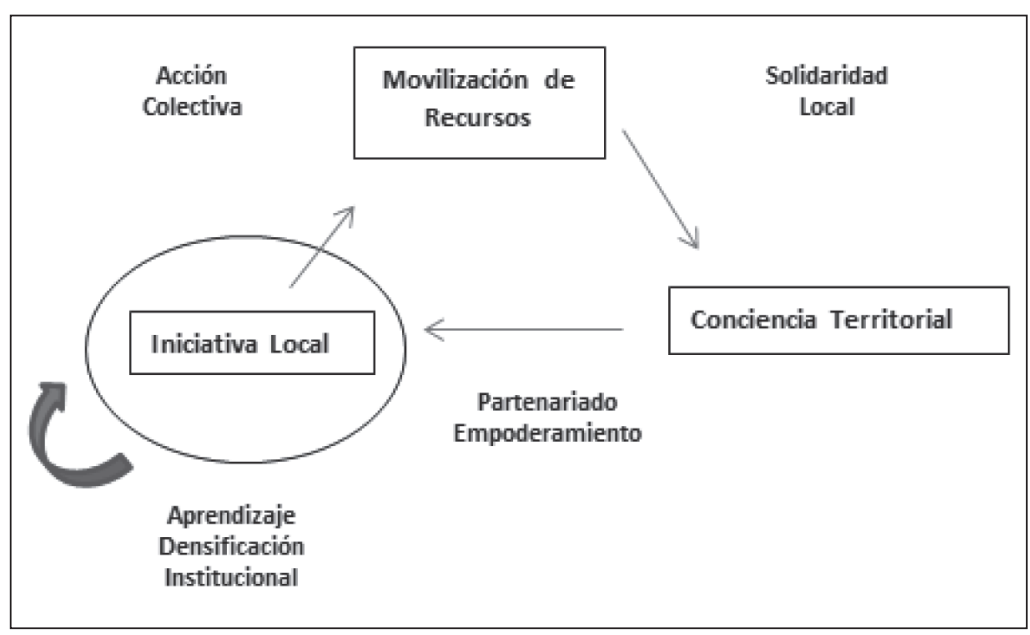

Fuente: Adaptado de Klein (2005).

\section{Análisis a las perspectivas de cada región}

Una solución a las estrategias de las políticas públicas para países de Latinoamérica es la que constituye la posibilidad de legitimar la acción de las instituciones sobre líneas de trabajo que condicionan la realidad del género en base a las políticas de oportunidades, acción positiva y la transversalidad (Arriagada, 2006). Esta institucionalidad de los gobiernos centrales en las políticas públicas debe visar a la descentralización de las acciones hacia problemas con demanda local regional (Arriagada, 2006; Guzmán, 2001). ¿Qué justificación es dada al 
poder público, cuando las reformas responden a administraciones que no manejan las políticas descentralizadas? Siendo de manera objetiva, neutral y técnica ¿es efectiva cuando es administrada por expertos ejecutivos en forma eficiente manteniendo la imparcialidad con el interés del género? (Guzmán, 2001). De acuerdo con Peterson (1995) y Handley (2008), la ineficiencia de administraciones por lo general no especifica resultados de un sistema en particular sino "un todo"; la entrega de un programa eficiente pasa por mejorar las relaciones entre gobiernos locales con la administración central. Por otro lado, también existen críticas para el sistema de administración descentralizada (federativa) que están asociadas al aspecto de gobierno que toca:
a) Envolvimiento relaciones locales - federal
b) Descentralización y política
c) Capacidad subnacional
d) Programa de responsabilidad
e) Programa de adaptabilidad.

La descentralización ciertas veces provoca una fragmentación en el alcance y extensión de las políticas y sus programas de servicios públicos al delegar responsabilidades para niveles de autoridad que se encuentran un poco abajo del gobierno central. Arriagada (2006) sucinta que debido a la fragmentación, se debe crear un marco de evaluaciones permanentes para corregir los impactos de las estrategias de las políticas sociales descentralizadas. Por ejemplo, lo que no puede ocurrir es que una administración deba responder por temas que corresponden a la unidad central mayor, es decir, el debate por temas que requieren grados de mayor atención y responsabilidad (en función de la situación de pobreza, situación de los centros de salud pública, desempleo estructural, etc.) también son de vital importancia para los gobiernos regionales.

La participación unida entre la formulación de las políticas y su implementación provoca y minimiza la fragmentación que provocan los programas descentralizados. De acuerdo con Handley (2008), en una política en proceso para programas federales es necesario evitar desconectar su andamiento entre niveles locales y federales, ya que afecta la vida de los ciudadanos locales cuando están siendo colocadas en práctica. La responsabilidad que los gobiernos requieren es de sumo esfuerzo de unidad política, ya que es necesario tomar en cuenta el impacto que provoca el cambio de comportamientos en un sistema federal sobre los sistemas de subvención a los programas de servicio público, que también pueden impactar en los gobiernos locales.

Por otro lado, es importante considerar la naturaleza del financiamiento, sea por programas de responsabilidad fiscal o ajuste en la política de gasto de los gobiernos locales. Los programas de servicio público requieren mantener a nivel central una estrechez en los niveles administrativos en cuales están siendo gestionados para nivel local Desarrollar un línea de gestión o administración horizontal es una estrategia de dialogo para evitar generar 
quiebres o producirlos de manera creativa generando nuevas oportunidades con otros enfoques de tratamiento (Christensen, 2001) principalmente cuando el tema es la asignación de recursos económicos para los programas de asistencia al empleo, o financiar programas de alcance productivo. La literatura sobre este aspecto menciona sobre lo esencial de conocer los principios que deben manejar los agentes acerca de la responsabilidad fiscal necesaria como además entenderla para una buena administración presupuestaria. Principalmente cuando existe una división jurisdiccional y la rigidez de una consciencia conservadora impide el uso de recursos con gastos que provienen desde agencias externa para financiar programas locales.

Los quiebres surgen debido a que los cambios, cuando no son completos por la falta de seguimientos o la falta de rigidez financiera en las evaluaciones permanentes, suelen ocurrir de manera transversal entre sectores de la administración central y local, que dejan vicios de manejo presupuestario, ya que muchas veces no existe control sobre la fuente de financiamiento externo para algunos programas de servicio público que no tiene un registro histórico. El rigor de un enfoque federal urge el control y la supervisión de dicha política fiscal local, por ejemplo el monitorear los programas del Consejo Nacional de la Mujer (CNM) en Argentina con las líneas locales de asignación de recursos en programas que son prioridad para las provincias. Para Guzmán (2001) esto se debe a la falta de una agenda de discusión sobre lo prioritario en la definición de los valores para un debate sobre la problemática del género en los programas de participación ciudadana.

De acuerdo con Agranoff y McGuire (2001) el sistema de subvención primaria a nivel de gobiernos locales presenta un modelo ejemplo dentro del federalismo en donde los gobiernos subnacionales se encuentran enfocados en mantener y dar cumplimiento junto al gobierno de la unión federal el control del sistema. Esto es evidente cuando los gobiernos locales presentan una carencia en general en la estrategia de administrar sus propios gobiernos, como puede ser poseer una legislación confusa, el solapamiento de programas para diferentes jerarquías, responsabilidad incierta, duplicación de esfuerzo administrativo para programas similares, etc. Las soluciones a estos tipos de situaciones son determinadas por el nivel federal al producir controles y supervisar los sistemas locales de administración.

\section{Orientación del liderazgo productivo para ambas regiones}

La conducción económica y la reproducción de liderazgos para conducir los programas institucionales de políticas de empleo a la mujer, requieren de mecanismos de producción que asuman la efectividad en el cumplimiento de los plazos propuestos. Ambos componentes son determinantes para lograr dicha efectividad, sin dejar de considerar la base ideológica en la emergencia y mantención de los programas sociales. Como el caso de los programas de corte productivo que articula el Servicio Nacional de la Mujer (Sernam) en Chile con las oficinas de la Mujer para cada administración local en conjunto con los actores del Gobierno en el Congreso Nacional. Para Blaug (2009) ciertamente no existe una relación entre la efectividad 
organizacional y la jerarquía, sino un proceso de sincronía independiente entre el trabajo humano individual y la colectividad en la búsqueda de prosperidad.

La razón está en que las organizaciones son dominadas por niveles jerárquicos que ciertamente son deliberadas por grupos hacia sus liderazgos. Y por conveniencia natural una organización se afirma sobre la capacidad del conocer y obtener información del entorno para mantención de la misma. Según la visión de las preferencias, hay un problema que surge en la toma de decisiones finales, con respecto al papel que desempeñan las políticas y los grupos de interés: el surgimiento de comportamientos personalizados con interés común, que puede inducir a políticas favorables, pero el hecho de revelar preferencias les otorga un individualismo suficientemente grande que afecta principalmente en la orientación de los programas y su propia asignación de recursos.

En este aspecto, cuando los grupos se constituyen en movimientos que apuntan hacia el bien común de sus metas, suele suceder que la jerarquía muchas veces tiende a corromper la participación democrática al reconocer los costos que incurren ciertos programas. Y finalmente se torna una cuestión ideológica que en último caso obstruye compartir el aprendizaje entre los grupos y empeora la convivencia. Por la misma razón es uno de los determinantes que hace aumentar los costos de inversión cuando se desconoce la conducción y no es claro el proceso del liderazgo del diseño de los programas sociales. Por ejemplo, para las democracias, la división jerárquica del trabajo político es legítima. Y según Blaug (2009) es necesaria dentro de sus representantes y ciudadanos cuando se detienen para ciertos cuestionamientos de conducción por parte de los agentes que lideran los programas.

Como estrategia de formulación e implementación puede ocurrir que la planificación del proceso esté mal enfocada o que para su definición requiera de un mayor esfuerzo colectivo, para dar claridad a los objetivos de las organizaciones. La fenomenología del trabajo de género es transversal en los valores y la literatura afirma que para organizaciones eficientes se deben preparar un plano de actividades que sean comprensibles de realizar para aquellos objetivos que han sido establecidos (Guzmán, 2001). La prospección de alternativas a programas sociales para emprendimientos requiere tener ideas y objetivos claros que ellos buscan, como elaborar ideas que estén en sintonía con los problemas y necesidades locales. Para una gestión estratégica de grupos de género, la autogestión y creación de nuevos emprendimientos va de la mano con la planificación y comprensión del entorno como el camino a maximizar la inversión en la entrega de servicios públicos (Bierbrauer, 2008).

Desde luego, el cuidado de la elección de los objetivos auxilia al desempeño del financiamiento (Boyd, 1991). Un estudio de Kelman y Myers (2009) para la innovación del sector público verificó que no hay una relación directa acerca si el éxito de innovaciones habían incluido avances en la forma de planificación de actividades a ser adoptadas para realizar innovaciones como objetivo de alcanzar diferentes resultados. La idea entre el desarrollo y una pequeña implementación durante el proceso de aplicación de los programas toma espacio de tiempo considerable para provocar un impacto y generar cambios en el método de los programas de trabajo (Golden, 1990). 
Para Krugman (1991) esta idea tiene como centralidad los obstáculos que pueden aparecer en la inercia de los gobiernos por la falta de competitividad y presiones que se generan en el desarrollo local de apalancar algunas regiones mas que otras (asimetrías en recompensas y falta de leyes para regular y controlar estos sistemas). Hay un aporte de aprendizaje en el ejemplo que producen las agencias de relativo éxito con sus programas de producción de manufactura local, que proyectan sus ideas hacia otras agencias de gobierno. Esto es, programas que aprovechan el contenido potencial de unas y lo aplican con éxito en otras organizaciones. Opuesto es lo que ocurre en el sector privado en la creación de cluster particulares propios de regiones que limitan recursos a estos formatos productivos. Quizás las políticas de gobierno para los formatos productivos locales de reconversión económica como salida laboral a la mujer, sean incursionar en programas de oportunidades, acciones positivas y dar transversalidad a través de sus gobiernos locales, como líneas estratégicas para el desarrollo local y que generen utilidad en gobiernos con una dispersión geográfica natural a través del país.

\section{Contribuciones del análisis de inversión a las iniciativas de productividad en programas sociales}

Para articular los elementos que promueven los programas de servicio público para la generación de empleo para la mujer, es preciso conocer cuáles son los elementos productivos y como estos se relacionan para cada región. Para los programas que proponen ambos casos en las regiones rurales de la Zona Central de Chile y la provincia de Santa Fe en Argentina, primero se elaboran una serie de informaciones que nos entregan una visión sobre la capacidad productiva desde el lado de la demanda de los programas que son necesarios incorporar, y por el otro lado lo que disponen los gobiernos como oferta pública para producir y elaborar el contenido de los programas que otorgan a las comunidades.

Este análisis plantea que existe una serie de diseños o modelos para la creación de programas que facilitan el entender sus dimensiones de atención social. Además los determinantes o elementos que incorporan los diseños son índices que figuran como relaciones óptimas para obtener buenos desempeños; toda vez que para la creación de modelos en los programas entregan alternativas al perfil de las inversiones que se realizan. Además de proponer un cuadro comparativo de algunos programas que pueden dar ciertas ideas acerca de qué determinantes generan un mejor resultado para evaluación que demandan retornos económicos a procesos de inversión (por ejemplo, algunos tomadores de precios que tienen comportamientos propios que pueden afectar el gasto público) y entender el impacto directo que provoca un mal diseño en la oferta de los programas sociales como también el impacto del seguimiento y evaluaciones de las políticas públicas en regiones.

Para lograr la articulación se propone un consolidado de programas que se desarrollan en ambas regiones. Tal vez es necesario comprender que el óptimo de soluciones a estos problemas está sustentado en la coordinación y preferencias de un buen servicio público. Para Gianakis (2002); Mensah, Schoderbek y Werner (2007); Hansman (1996) el empleo de 
una serie de elementos motivadores, hacen que las instituciones del sector público sean más eficientes, más efectivas y den sustento a una gestión pública responsable por el resultado de sus políticas públicas.

Siendo la búsqueda de empleo el problema general, el fenómeno en estudio es el análisis de los programas de reconversión económica para el género. Para efectos de visualización de entradas y egresos como resultados de políticas, se establecen algunas variables que pueden ser utilizas como instrumentos que amenazan el buen desempeño de lo que podrían ser los programas. Este análisis incorpora una parametrización de cómo los elementos en el contenido de los programas se relacionan para entregar un óptimo como resultado a procesos de inversión y mejorar la eficiencia. Por ejemplo, en una ecuación en lo cuadro 1 es una relación positiva ya que se propone evaluar un programa que tenga un valor $\lambda^{1}$ que se puede asociar al incremento en el valor de los costos de su asistencia social y entender lo que es más conveniente al momento de evaluar, también lo mismo sucede para una función con orientación hacia resultados ya que $\lambda^{2}$ se asocia a resultados crecientes. A partir de ambos tipos de parametrizaciones 1 y 2 con sus elementos o determinantes de control X-Y relacionados a otro determinante $\mathrm{Z}$ exógeno podemos inferir que no está sujeta a controles de gestión o administración (por ejemplo, precios). La ecuación (1) es exógena con respecto a la ecuación (2) ambas puedes ser entendidas como un cruzamiento de variables que permiten hacer efectivo la relación de costo y entender algunas variables como instrumentos de control. Así como acotar la toma de decisiones en función del aumento o disminución del gasto o la política fiscal de cada gobierno. Mensah, Schoderbek y Werner (2007), Hansmann (1996) sostienen que las buenas instituciones aceptan criterios de análisis multidimensionales que son necesarios para evaluar el desempeño de acuerdo a las perspectivas de quienes reciben los beneficios.

Por otra parte, es crucial observar que la implementación de instrumentos participativos de gestión innovadores para estos formatos de producción local se tornan efectivos cuando sus réplicas entregan buenos resultados en otras regiones. Un cuadro comparativo de políticas públicas transversales de intervención del género es útil a modo de generar resultados cualitativos y cuantitativos, de manera de producir plataformas seminales para otros programas de participación ciudadana, que permiten crear y desarrollar controles de medición y puntos de riesgos (cuadro 2, representación al parametrización las Funciones y sus elementos y variables).

\section{Cuadro 1}

Considerar la siguiente ecuación como herramienta de control y conducción con potencial endógeno para ambos casos

$$
\begin{aligned}
& Y 1=a 0+(a 1 X 1+n .+a n X n+a n+1 Y 2)+(\lambda 1 Z 1+\ldots \lambda m Z m)(1) \\
& Y 2=c 0+(b 1 W 1+n+b s W s+b s+1 Y 1)+(\gamma 1 Z 1+\ldots . \gamma m Z m)(2)
\end{aligned}
$$

Fuente: Adaptado de autores. 
Cuadro 2

\section{Representación parametrizada e implicancia de las variables y elementos} según el tipo de orientación de gestión

\begin{tabular}{|c|c|c|c|c|c|c|}
\hline Tipo Variable & Costo & Escala & Resultado & Variables del Entorno $(\mathrm{x})$ & Implicancia del Entorno (W) & Orientacion Gestion ( $\mathrm{Z})$ \\
\hline $\mathrm{X}_{1} ; \mathrm{W}_{1} ; \mathrm{Z}_{1}$ & $Y_{1}$ & $1-100$ & Y2 & Capital Economico & $\begin{array}{l}\text { Transporte, Infraestructura Comunicacional, Espacio, } \\
\text { Vinculos Economicos, Inversion Privada, Background Business }\end{array}$ & $\begin{array}{l}\text { Precio, Mercado, } \\
\text { Servicio; Productos }\end{array}$ \\
\hline $\mathrm{X}_{1} ; \mathrm{W}_{1} ; \mathrm{Z2}$ & $Y_{1}$ & $1-100$ & $\mathrm{Y} 2$ & Capital Humano & $\begin{array}{l}\text { Actitud, Confianza, Grado de Emprendimientos, Riesgo, Grados } \\
\text { Destreza }\end{array}$ & $\begin{array}{l}\text { Precio, Mercado, } \\
\text { Servicio; Productos }\end{array}$ \\
\hline $\mathrm{X}_{1} ; \mathrm{W}_{1} ; \mathrm{Z3}$ & $Y_{1}$ & $1-100$ & Y2 & Capital Social & $\begin{array}{l}\text { Vinculos Redes Publica, Privado y Tercer Sector, Calidad Institucional } \\
\text { y Gobiernos locales, normas y facilidad cooperacion de grupos. }\end{array}$ & $\begin{array}{l}\text { Precio, Mercado, } \\
\text { Servicio; Productos }\end{array}$ \\
\hline $\mathrm{X}_{1} ; \mathrm{W}_{1} ; \mathrm{Z4}$ & $Y_{1}$ & $1-100$ & Y2 & $\begin{array}{l}\text { Capital Cultural } \\
\text { Consenso Politico }\end{array}$ & $\begin{array}{l}\text { Compromiso Civil; Fomento de Politicas; Identidad, Sentido del } \\
\text { Espacio Publico, Valorizacion Cultural. }\end{array}$ & $\begin{array}{l}\text { Precio, Mercado, } \\
\text { Servicio; Productos }\end{array}$ \\
\hline
\end{tabular}

Fuente: Adaptado de autores.

Donde: $\mathrm{Y} 1$ = Costos; $\mathrm{Y} 2$ = Resultados; $\mathrm{X} 1 \ldots \mathrm{Xn}=$ Variables exógenas que determinan los tipos de costos. W1 ...Ws $=$ Variables exógenas que determinan los niveles de resultados; $\mathrm{Z1} \ldots \mathrm{Zm}=$ Variables de gestión que afectan tanto los costos como los tipos de resultados

La equidad de género es un tema relevante en cuanto a las oportunidades que proyecta y a la necesidad que se revela para las regiones, siendo aún un concepto que ha tomado fuerza a nivel global en la última década. Principalmente tiende a constituir nuevas organizaciones con actores que requieren programas de relevancia con foco en la productividad y desarrollo local para la mujer. A pesar que existen enfoques tradicionalistas de mantención por asistencialismos y otros que matizan incorporar la nueva economía del conocimiento como factor de relevancia a los programas sociales, el enfoque será positivo en cuanto no afecte los requerimientos que comparten las comunidades del género para la mujer en cuanto a la integridad cultural, sus valores o su identidad en los formatos productivos donde desean emprender sus actividades (Adkins, 1995, 1999; Lash 1994; Banks y Milestone, 2011; Perrons, 2007; Guzmán, 2001).

\section{Aplicaciones y elementos determinantes en los programas sociales}

En el contexto regional, la equidad de género se torna un tema estratégico dentro de una agenda de política social de gobiernos que incluyen el aspecto étnico-racial, etario, demográfico, geográfico, discapacidad y otras formas relacionadas a la discriminación de salidas al empleo. La preocupación que levantan ciertos cuestionamientos es entender cómo responden al desempeño las empresas y la conductas de mercado frente a las diversas trayectorias que toman los entornos de esta aglomeración de regiones y sus componentes estratégicos frente a la globalización (Terluin, 2003; Porter, 2000); principalmente la búsqueda de fuentes de recursos para apalancar las oportunidades del género.

La política según Baskin (2006) es muy similar a un sistema de no discriminación parecido en su parcialidad a un plan elaborado que entrega igualdad de oportunidades en políticas laborales para empresas que son globales. De acuerdo con Courtney y Moseley (2008) 
tanto el desarrollo de factores tangibles — - v.g la inversión pública- como elementos menos tangibles — v.g. la capacidad institucional— son inductores del éxito económico, al considerar que existen vínculos de factores de mayor complejidad que están enraizados en un contexto local socio económico, histórico y cultural. Ante un contexto de estas dimensiones, Courtney y Moseley (2008) identificaron algunas dimensiones que denotan ciertos grados de desarrollo y desempeño local en regiones rurales bajo circunstancias de desempleo y escasos ingresos, desplazamientos demográficos y búsqueda de empleo, privación y comienzo de nuevos negocios. Según el enfoque sectorial que poseen ambas regiones es posible constatar una menor complejidad de relaciones entre estos factores mencionados que permiten explicar diferentes desempeños en un contexto de la variedad espacial que poseen hacia el desarrollo local con perspectivas de salidas laborales al género.

El cuadro 3 es una matriz sectorial que incorpora las 4 dimensiones sobre algunas nociones que deben considerarse en el contenido de las propuestas para promover el desarrollo local y el fomento de algunas líneas de inversión para propuestas de colocación de trabajo. Además de entender la dinámica del desempeño de programas de reconversión económica de mano de obra como política transversal sectorial. La ocupación de la mujer podrá darse en diferentes sectores de la economía, principalmente en aquellos que demandan alto grado de instrucción, profesionales liberales; que de acuerdo al contexto de alto desempeño estas actividades per se, atraerán mayores ingresos (Shire, 2007; Walby, 2006, 2011). Pero esto no se traduce de manera directa en movilidad en jerarquía como resultado de esta dinámica de procesos productivos (Gerson, 2004; Clark, 2000; Emslie y Hunt, 2009; Guest, 2002).

El estímulo endógeno al crecimiento local induce a los gobiernos centrales dar mayor atención de fomentar las ideas como economía del conocimiento, generar alianzas con otros gobiernos vecinos, apoyar la correcta planificación de políticas locales, identificar redes de trabajo en un contexto del género (McGuire, 2000), con el objetivo de dar soporte al desarrollo del entorno.

\section{Cuadro 3}

\section{Marco dimensional para desarrollo local para grupos de fomento y fuentes de elementos}

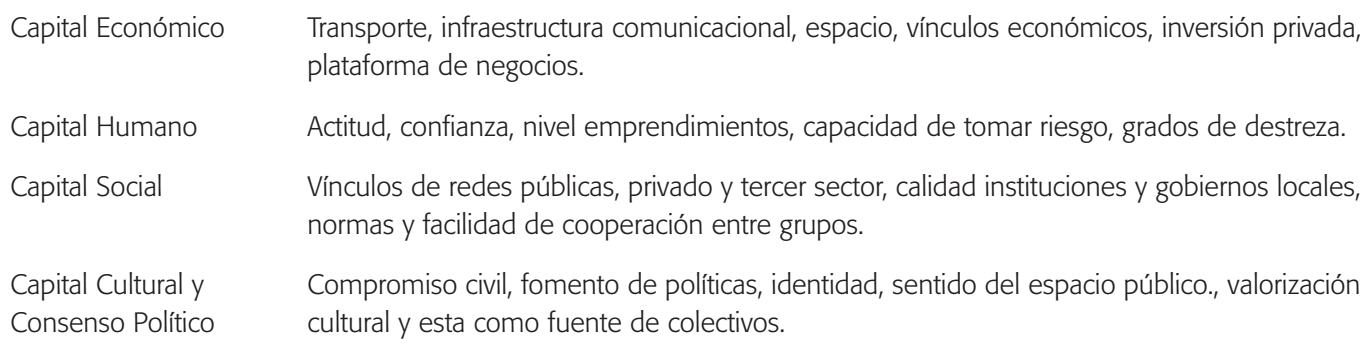

Fuente: Adaptada de (Courtney y Moseley, 2008). 
El cuadro 3 confirma las relaciones entre el diseño estructurado de las relaciones dimensionales como base previa a la formulación de programas de desarrollo y fomento a grupos de economía local. Formalmente la representación de las fuentes permite reflejar la interacción de redes que pueden construirse en función de conductas y comportamientos de una oferta de fuerza laboral con fuertes contrastes en la base de una sociedad de conocimiento para ambas regiones, por ejemplo, para el caso de la fuerza de trabajo se constata que el mercado laboral alcanza en Chile solo al 42,3\% de las mujeres y la tasa de indigencia y desocupación alcanza $54,6 \%$, encontrándose pobres-desocupadas el 35,9\%.

El aspecto dimensional induce a una oferta de oportunidades en la constitución de firmas y emprendimientos con base a una demanda social. En el caso de las zonas rurales de la Zona Central de Chile para el año 2010 la intervención según el estudio Fosis (2010) a los emprendimientos formales era de 10,7\% y su informalidad de 83,8\% en el momento de intervención de los programas, 85\% están en el tramo de 24-59 años; y el nivel de empleabilidad están en el tramo de 19-24 años que corresponde al 68\%. Es importante resaltar que los beneficios sociales de esta interacción deben estar bajo un soporte institucional que prima por el resultado de las interacciones de grupos de trabajo, que lejos de la informalidad como fuente de creación de redes de trabajo, envuelven una seria de opciones paternalistas de apoyo a estos otros emprendimientos, visto como un encadenamiento de producción tradicional distante de un modelo económico para igualdad del género la flexibilidad de nuevas firmas.

Mientras tanto, los modelos figuran mucho mas para una individualización frente a la falta de opciones a favor del empleo individual (Beck, 1992; Beck y Beck-Gernsheim, 2002). Los modelos de contenido que demandan los programas al parecer están asentados sobre la creación de redes de emprendimientos sociales con fuertes perspectivas de construcción de una economía de conocimientos y tipos de organizaciones con alto grado de diferenciación entre las relaciones formales e informales que afectan estos tipos de formatos de organizaciones con enfoque del género (Van Emmerik et al., 2006; Durbin, 2011; Blau, 1967:112). Para el caso Chileno la edad promedio de escolaridad de mujeres que participan en estos tipos de emprendimientos es de 10,3 años de escolaridad. Sin embargo la participación laboral femenina en Latinoamérica alcanza al 63\% en Argentina y un 52.2\% en áreas urbanas, de esta misma, las ocupaciones dominadas por la función genero con participación de la mujer se identifican en el área Pedagógica 72.7\%; Textil 82.0\%, Ayuda domestica 86.4\%, Cuidado personal 88.5\%, Secretarias 92.1\%. Por otro lado la participación en emprendimientos por cuenta propia al año 2009 según el estudio de la Casen en Chile era 19.2\%, un 51.4\% participaba en el sector privado, $12 \%$ desempeñaba labores domésticas; $14.1 \%$ en el sector público. De lo anterior, vemos que es necesario entender qué tipos de componentes dimensionales tiene la relación de diferenciación que permita fomentar estos tipos de formatos entre hombres y mujeres, por medio de redes de trabajo que animen un colectivo de aprendizaje mutuo (Bowen, 2001; Guillaume y Pochic, 2009). 


\section{Cuadro 4 \\ Diseño de programas desarrollo local con enfoque del Género en Zona Central de Chile y la Provincia de Santa Fe (Argentina)}

\section{PROGRAMAS SOCIALES \\ ZONA CENTRAL \& PROVINCIA SANTA FE}

Políticas de Conciliación y Corresponsabilidad (Familia- Demanda doble responsabilidad por trabajo remunerado Trabajo) y Órganos Públicos que fomentan los programas y no remunerado, política relacionada con el embarazo y maternidad.

A) Sistema de Protección Social con Perspectiva de Género hacia la Mujer

(1) Chile Solidario (Mideplan)

(2) Chile Crece Contigo (Mideplan)

(3) Puente (Fosis)

(4) Programa Apoyo al Microemprendimiento (Fosis)

(5) Programa Emprende Más (Fosis)

(6) Programa Empleabilidad Juvenil (Fosis)

(7) Programa Preparación Nivelación y Competencia Laboral (Fosis)

B) Plan nacional Integral Manos a la obra (Aportes del Ministerio de Desarrollo Social de la Nación a los Gobiernos Provinciales. Programa que evalúa propuestas a través de una unidad evaluadora de la Dirección Provincial de Desarrollo territorial y Economía social de la Provincia de Santa Fe, compuesta por miembros de Gobiernos Nacionales y Provinciales.)

C) Programa Provincial de Subsidios para Emprendedores productivos sociales

(Dirección Provincial de Desarrollo territorial y Economía social de la Provincia de Santa Fe, dependiente del Min. de Desarrollo Social de dicha provincia. Intervención provincial pura)

\section{OBJETIVOS}

Política que genera estrategias e implementa medidas que acompaña el ciclo de vida de las personas, promueve capacidad generadora de ingreso individual y familiar.

(1) enfoca la capacidad de generación de ingresos y transferencia monetaria, como mecanismo de superación de extrema pobreza, identificar nichos e inclusión en redes sociales en mercados laborales, producción de autoconsumo.

(2) enfatiza responsabilidad equitativa del cuidado parental integral de menores.

(3) promueve tareas productivas y reproductivas, reconocimiento explicito de roles en hogares monoparentales, apoyo psicosocial.

(4) capacitación y transferencia monetaria para mejorar actividad económica, mejora de ingresos.

$(5,6,7)$ capacitación y aporte monetario.

Intervenciones tendientes al fortalecimiento de cadenas asociativas y/o productivas de emprendimientos que contribuyan al desarrollo local de las regiones.

Se sitúan los grupos familiares más vulnerables del tejido social. Detectada en ellos, alguna destreza o habilidad en el campo de las producciones, considera la recuperación de estos saberes o conocimientos, como punto de partida para acceder progresivamente, a niveles más complejos de producción. Intervenciones que fortalecen los vínculos interpersonales e interfamiliares, construyendo redes de trabajo en los territorios entre las diferentes organizaciones e instituciones en cada uno de estos. 


\begin{tabular}{|c|c|}
\hline $\begin{array}{l}\text { PROGRAMAS SOCIALES } \\
\text { ZONA CENTRAL \& PROVINCIA SANTA FE }\end{array}$ & OBJETIVOS \\
\hline $\begin{array}{l}\text { D) Programa Banco Solidario } \\
\text { (Ministerio de Desarrollo Social de la Provincia de Santa } \\
\text { Fe) }\end{array}$ & $\begin{array}{l}\text { Se orientan a o subsidios no reintegrables otorgados a Organizaciones } \\
\text { administradoras (organizaciones de la sociedad civil, etc.). } \\
\text { Los beneficiarios son pequeños emprendedores cuya condición } \\
\text { de vulnerabilidad social, no les posibilita acceder a otra línea } \\
\text { de créditos. }\end{array}$ \\
\hline $\begin{array}{l}\text { E) Programa "Los nuevos oficios" } \\
\text { (Dirección Provincial de Desarrollo territorial y Economía } \\
\text { social de la Provincia de Santa Fe) }\end{array}$ & $\begin{array}{l}\text { Sistematización mensual de espacios de encuentros jóvenes } \\
\text { de diferentes Ministerios, Secretarías, Direcciones y áreas de } \\
\text { trabajo, intercambiar experiencias y opiniones, que posibiliten } \\
\text { la inclusión social de jóvenes y adultos al trabajo. } \\
\text { A escala territorial, propone la inclusión de los y las jóvenes } \\
\text { en los ámbitos de decisión y el fomento de producciones } \\
\text { innovadoras. }\end{array}$ \\
\hline
\end{tabular}

Fuente: Elaboración propia de los autores.

La capacidad que manejan los grupos que se componen de mujeres parece ser un fenómeno que fortalece el énfasis de fomentar la creación de organizaciones emprendedoras, sea por el desarrollo que premian la construcción de estos formatos por parte de los gobiernos o por implementar nuevos enfoques de un aprendizaje tecnológico para un desarrollo global (Novy-Hidesley, 2006). Sin embargo esta relación no es directa en el incremento de nuevas salidas laborales o igualdad, que podría estar vinculada en un mayor grado de instrucción en los grupos del género cuando el enfoque es considerado de capital tecnológico (Caprile y Pascual, 2011) o producir un aumento vertical en las funciones de alto rango y mantener un buen empleo relacionado con un alto ingreso (Guillaume y Pochic, 2009).

Los diversos enfoques que puede ofrecer la exposición natural de algunos entornos locales como poseen algunos sectores que conglomeran tecnologías y manufactura pueden dar algunas ideas hacia donde deben orientarse los programas sociales. Si bien el aspecto tecnológico es una plataforma diversa para creación de nuevos emprendimientos, requieren asumir un riesgo considerado al demandar determinados niveles de inversión. La exposición de organizaciones informales, sobre todo en emprendimientos que poseen ciertos elementos de innovación, pareciera ser mucho más enriquecedoras cuando estos formatos particulares marcan su propia identidad (Mósesdóttir, 2006, 2011; Castells, 1996, 2001; Chasseiro y Legault, 2010; Lawler, 2008).

\section{Aspectos conclusivos}

Ambas regiones destinan parte sus políticas públicas a elaborar y formular programas que demandan un interés global que consiste en atender a sectores marginalizados en la estructura socio-económica. La participación de grupos organizados en ambas regiones es notada por la 
orientación al desarrollo de formatos productivos locales, visto desde la formación organizaciones emprendedoras.

El foco del género en ambas regiones se manifiesta con diferente intensidad. En el caso de la provincia de Santa Fe, no se observan programas sociales de fomento productivo que pongan el foco en la mujer como elemento central en si misma. No obstante, se observa un eje mucho más amplio, que implica considerar al grupo familiar como beneficiario de estas políticas. Esto no quiere decir que la cuestión de género se encuentre ausente de la agenda del gobierno santafesino, sino que la misma tiene su mayor relevancia en los aspectos de carácter social de la mujer. De hecho destacamos la existencia de organismos gubernamentales a nivel provincial, como lo es la Dirección Provincial de las Mujeres, que promueve la igualdad de condiciones en el campo educativo, laboral, de salud, de vivienda, cultural y deportivo.

Por otra parte se observa que la atención de la mujer en la Zona Central es mayor que en Santa Fe. Y vale arriesgar decir que la atención de Programas para Jóvenes y Adultos parece ser de mayor urgencia en la comunidad santafesina que en la Zona Central de Chile. En fin, se trata de políticas públicas con diferentes alcances de género desde la perspectiva de la mujer. Unos enfocan mayormente hacia el aumento del ingreso familiar, siendo actor principal la mujer joven y adulta. Y en otro caso, el eje lo constituye la reconversión económica de la mano de obra laboral en la población, privilegiando mayormente el ingreso (o reingreso) del hombre joven adulto al mundo laboral.

Si bien los programas del género destinados a la Zona Central presentan fuerte transversalidad de temas que afectan tanto a mujer como hombre, son temas que reproducen el interés principal en producir formatos organizacionales emprendedores, principalmente en aquellos donde la mujer no percibe ingresos por si sola. Debido a que los programas tiene un fuerte fomento estatal, esto genera un problema en la base de la política económica que afecta al gasto público por la forma de cómo se hacen sostenibles estos sistemas organizacionales a largo plazo. Sumado a lo anterior, se observó en ambos casos que el Gobierno Central proporciona el financiamiento de los programas a través de las transferencias monetarias de ayuda o fomento publico a promover capacidades y organizar formatos productivos.

Como aspecto final de cada región, se observa la existencia de cierta independencia en producir líneas estratégicas de desarrollo local que orientan hacia donde las políticas publicas deben sentar sus objetivos. El liderazgo (político, económico o social) es una instancia oportuna para que cada región logre mantener intacto el contenido de sus propios programas, aprovechando el marco institucional que las cobija, permitiendo la participación a nivel local en la discusión de los programas sociales. No obstante, la posibilidad de que estos liderazgos cambien en pleno "tránsito" el desarrollo de los programas sociales, constituye un riesgo de que las variables de medición y los costos incurran en grandes perjuicios económicos para cada una, independiente del alcance que se pretende realizar. 


\section{Referencias}

ABRUCIO, Fernando. A coordenação federativa no Brasil: A experiência do período FHC e os desafios do governo Lula. Revista de Sociologia e Política, v. 24, n. 1, p. 31-67, 2005.

ADKINS, Lisa. Community and economy: a retraditionalization of gender? Theory, Culture and Society Journal, v. 16, n. 1, p. 117-137, 1999.

ADKINS, Lisa. Gendered work: sexuality, family and the labour market. Buckingham: Open University Press, 1995.

AGRANOFF, Robert; MCGUIRE, Michael. American federalism and the search for models of management. Public Administration Review, v. 61, n. 1, p. 671-681, 2001.

ARRETCHE, Marta. Federalismo e políticas sociais no Brasil: problemas de coordenação e autonomia. São Paulo em Perspectiva, v. 18, n. 2, p. 17-26, 2004.

ARRIAGADA, Irma. Cambios de las políticas sociales: políticas de género y familia. Cepal - Serie Políticas sociales, n. 119, abril, 2006.

BANKS, Mark; MILESTONE, Katie. Individualization, gender and cultural work. Gender, Work and Organization Journal, v. 18, n. 1, p. 73-89, Jan. 2011.

BASKIN, Jeremy. Corporate responsibility in emerging markets. Journal of Corporative Citizenship, v. 19, n. 24, p. 29-47, 2006.

BECK, Ulrich. Risk society. London: Sage, 1992.

BECK, Ulrich; BECK-GERNSHEIM, Elizabeth. Individualization. London: Sage, 2002.

BIERBRAUER, Felix. A unified approach to the revelation of public goods preferences and to optimal income taxation. Max Planck Institute for Research on Collective Goods, Bonn, n. 39, p. 1-36, Oct. 2008.

BINZ-SCHARF, Maria; LAZER, David; MERGEL, Ines. Searching for answers: networks of practice among public administrators. RWP08-046, HKS Faculty Research Working Paper Series, Oct. 2008.

BLAU, Peter. Exchange and power in social life. London: John Wiley \& Sons, 1967.

BLAUG, Ricardo. Why is there hierarchy? Democracy and the question of organizational form'. Critical Review of International Social and Political Philosophy, v. 12, n. 1, p. 85-99, 2009.

BOWEN, Rene. Developing relational practices for knowledge intensive organizational contexts. Career Development International, v. 6, n. 7, p. 361-369, 2001.

BOYD, Brian. Strategic planning and financial performance: a meta-analytic review. Journal of Management Studies, v. 4, n. 28, p. 353-374, 1991.

BUCHANAN, James. The Coase theorem and the theory of the State. In: BUCHANAN, James; TOLLISON, Robert (Org.). The public choice II. Ann Arbor, University of Michigan Press, 1999. p. 159-173. 
CAPRILE, Maria; PASCUAL, Amparo. The move towards the knowledge-based society: a gender approach. Gender, Work and Organization, v. 18, n. 1, p. 48-72, Jan. 2011.

CASTELLS, Manuel. The internet galaxy: reflections on the internet business and society. New York: Oxford University Press, 2001.

CASTELLS, Manuel. The rise of the network society. The information age: economy, society and culture. Oxford: Blackwell, 1996.

CHASSEIRO, Stéphanie; LEGAULT, Marie-Josée. Discretionary power of project managers in knowledge-intensive firms and gender issues. Canadian Journal of Administrative Sciences, v. 27, n. 1, p. 236-248, 2010.

CHILD, John; MCGRATH, Rita G. Organizations unfettered: organizational form in an informationintensive economy. Academy of Management Journal, v. 44, n. 6, p. 1135-1148, 2001.

CHRISTENSEN, Tom. Administrative reform: changing leadership role? International Journal of Policy and Administration, v. 14, n. 4, p. 457-480, 2001.

CLARK, Sue C. Work-family border theory: a new theory of work-family balance. Human Relations, v. 53, n. 6, p. 747-70, June 2000.

COURTNEY, Paul; MOSELEY, Malcom. Determinants of local economic performance: experience from rural England. Local Economy, v. 23, n. 4, p. 305-318, Nov. 2008.

CRANDALL, Christian; BEASLEY, Rian. A perceptual theory of legitimacy. In: JOST, John; MAJOR, Brenda (Ed.). The psychology of legitimacy: emerging perspectives on ideology, justice, and intergroup relations. Cambridge: Cambridge University Press, 2001. p. 77-102.

DURBIN, Susan. Creating knowledge through networks: a gender perspective. Gender, Work and Organization, v. 18, n. 1, p. 90-112, Jan. 2011.

EMSLIE, Caroline; HUNT, Kane. Live to work or work to live? A qualitative study of gender and work-life balance among men and women in mid-life. Gender, Work and Organization, v. 16, n. 1, p. 151-172, Jan. 2009.

FERNANDEZ, Sergio; RAINEY, Hal. Managing successful organizational change in the public sector: an agenda for research and practice. Public Administration Review, v. 66, n. 2, p. 168-176, Mar./ Apr. 2006.

GERSON, Kathleen. Understanding work and family through a gender lens. Community, Work and Family, v. 7, n. 2, p. 163-178, Aug. 2004.

GIANAKIS, Gerasimos. The promise of public sector performance measurement: anodyne or placebo? Public Administration Quarterly, v. 26, n. 3, p. 35-64, 2002.

GOLDEN, Olivia. Innovation in public sector human services programs: the implications of innovation by 'groping along'. Journal of Policy Analysis and Management, v. 9, n. 2, p. 219-248, 1990.

GRANT, Adam. Does intrinsic motivation fuel the prosocial fire? Motivational synergy in predicting persistence,performance, and productivity. Journal of Applied Psychology, v. 93, n. 1, p. 48-58, 2008. 
GUEST, David. Perspectives on the study of work-life balance. Social Science Information, v. 41, n. 2, p. 255-279, 2002.

GUILLAUME, Cécile; POCHIC, Sophie. What would you sacrifice? Access to top management and the work--life balance. Gender, Work and Organization, v. 16, n. 1, p. 14-36, Jan. 2009.

GUZMÁN, Virginia. La institucionalidad del genero en el Estado: nuevas perspectivas de análisis. Cepal — Serie Mujer y desarrollo, LC/L.1511-P, Santiago de Chile, mar. 2001.

HANDLEY, Donna M. Strengthening the intergovernmental grant system: long-term lessons for the federal-local relationship, Public Administration Review, v. 68, n. 1, p. 110-125, Jan./Feb. 2008.

HANSMANN, Henry. The ownership of enterprise. Cambridge MA: Belknap Press, 1996.

KELMAN, Steven. Changing big government organizations: easier than meets the eye? RWP04-026, KSG Faculty Research Working Paper Series, May 2004.

KELMAN, Steven. Downsizing, competition, and organizational change in government: is necessity the mother of invention. Journal of Policy Analysis and Management, v. 25, n. 4, p. 875-895, 2006.

KELMAN, Steven; MYERS, Jeff. Successfully executing ambitious strategies in government: an empirical analysis. RWP09-009, HKS F.R. Working Paper Series, Apr. 2009.

KLEIN, Juan Luis. Iniciativa local y desarrollo: respuesta social a la globalización neoliberal. Revista Eure, v. 31, n. 94, p. 25-39, Dic. 2005.

KRUGMAN, Paul. Geography and trade. Cambridge, MA: MIT Press, 1991.

KUVAAS, Bard; DYSVIK, Anders. Perceived investment in employee development, intrinsic motivation and work performance. Human Resource Management Journal, v. 19, n. 3, p. 217-236, 2009.

LASH, Scott. Reflexivity and its doubles: structure, aesthetics, community. In: BECK, Ulrich; GIDDENS, Anthony; LASH, Scott. Reflexive modernization. Cambridge: P. Press, 1994. p. 110-173.

LAWLER, John. Individualization and public sector leadershi. Public Administration Journal, v. 86, n. 1, p. 21-34, 2008.

LOCKE, John. The second treatise of government. Indianapolis: Bobbs-Merrill, 1952.

MACHADO, José Angelo. Gestão de políticas públicas no Estado federativo: apostas e armadilhas. Dados — Revista de Ciências Sociais, Rio de Janeiro, v. 51, n. 2, p. 433-457, 2008.

MCGUIRE, Gail. Gender, race, ethnicity and networks: the factors affecting the status of employees' network members. Work and Occupations Journal, v. 27, n. 4, p. 501-524, 2000.

MELAMED, Claire. Caught in the crossfire: the economic crisis and developing Countries. Public Policy Research, v. 16, n. 1, p. 45-50, Mar./May 2009.

MENSAH, Yaw; SCHODERBERK, Michael; WERNER, Robert. A methodology for evaluating the costeffectiveness of alternative management tools in public-sector institutions: an application to public education. Disponible en: <http://ssrn.com/abstract=899401>. Acceso en: Jan. 2007. 
MÓSESDÓTTIR, Lilja. Gender (in) equalities in the knowledge society. Gender, Work and Organization, v. 18, n. 1, p. 30-47, Jan. 2011.

MÓSESDÓTTIR, Lilja. Introduction: tackling employment and gender challenges. In: MÓSESDÓTTIR, Lilja; REMERY, Chantal; PASCUAL, Amparo S. (Org.). Moving Europe towards the knowledge based society and gender equality: policies and performances. Brussels: ETUI. 2006. p. 1-21.

NOVY-HILDESLEY, Julia. From idea to impact funding invention for sustainability: innovations case discussion: kickstart. Innovations: Technology, Governance, Globalization, v. 1, n. 1, p. 31-42, 2006.

OATES, Wallace. An essay on fiscal federalism. Journal of Economic Literature, v. 37, n. 3, p. 11201149, 1999.

PEP. Plan Estratégico de la Provincia de Santa Fe, 2008. Disponible en: < http://www.santafe.gov. ar/index.php/web/guia/gobernador?cat=plan_estrategico $>$.

PEP. Plan Estratégico de la Provincia de Santa Fe, 2008. Disponible en: < http://www.santafe.gov. ar/index.php/web/content/view/full/65147>.

PERRONS, Diane. Living and working patterns in the new knowledge economy. In: WALBY, Sylvia et al. (Org.). Gendering the knowledge economy. London: Palgrave, 2007. p. 188-206.

PERSSON, Torsten; ROLAND, Gerard; TABELLINI, Guido. Separation of powers and political accountability. Quarterly Journal of Economics, v. 112, n. 4, p. 1163-1202, 1997.

PETERSON, Paul. The price of federalism. Washington, DC: Brookings Institute, 1995.

PORTER, Michael. The competitive advantage of nations. London: Macmillan Press, 2000.

PUTNAM, Robert. Bowling alone. The collapse and revival of American community. New York: Simon and Schuster, 2003.

PUTNAM, Robert. Making democracy work: civic traditions in modern Italy. New Jersey: Princeton University Press, 1993.

PYKE, Frank; BECCANTINI, Giacomo; SENGENBERGER, Werner. Industrial districts and inter-firm co-operation in Italy. Geneva: International Institute for Labor Studies, 1990.

SHIRE, Karen. Gender and the conceptualization of the knowledge economy in comparison. In: WALBY, Sylvia et al. (Org.). Gendering the knowledge economy. London: Palgrave, 2007. p. 51-77.

SIMANI, Erick; HART, Stuart. Expanding possibilities at the base of the pyramid. Innovations Journal, v. 1, n. 1, p. 43-51, 2006.

STIVERS, Camila. Bureau men, settlement women: constructing public administration in the progressive era. Lawrence: University Press of Kansas, 2000.

STIVERS, Camila. The significance of the administrative State. Public Administration Review, v. 68, n. 1, p. 53-56, Jan./Feb. 2008.

TERLUIN, Ida. Differences in economic development in rural regions of advanced countries: an overview and critical analysis of theories. Journal of Rural Studies, v. 19, n. 3, p. 327-344, 2003. 
TUROK, Ivan. The SNP government and poverty. Local Economy Review, v. 23, n. 4, p. 255-260, 2008.

VAN EMMERIK, Hetty et al. Networking your way through the organization: gender differences in the relationship between network participation and career satisfaction. Women in Management Review, v. 21, n. 1, p. 54-66, 2006.

WALBY, Sylvia. Is the knowledge society gendered? Gender, Work and Organization, v. 18, n. 1, p. 1-29, Jan. 2011.

WALBY, Sylvia. The gendered knowledge economy in the UK. Lancaster University and Institute of Social Science; University of Tokyo, 2006.

WALDO, Dwight. The administrative State: a study of the political theory of American public administration. New York: Ronald Press, 1948.

Cristian Eugenio Orellana Pino, ingeniero en administración de empresa mención marketing, licenciado en ciencias de la administración, Facultad de Economía y Empresa por la Universidad Diego Portales (UDP), Santiago de Chile. E-mail : cristian1914@hotmail.com.

Hernán José Perotti, contador público y especialista en contabilidad y auditoría por la Facultad de Ciencias Económicas de la Universidad Nacional del Litoral (Santa Fe — Argentina). Docente e investigador del Departamento de Contabilidad e Impuestos de la FCE-UNL. E-mail: hernanperotti@yahoo.com.ar. 